\title{
Challenges in testicular cancer management
}

\author{
Joel Sheinfeld
}

Received: 5 July 2009 / Accepted: 5 July 2009 / Published online: 16 July 2009

(C) Springer-Verlag 2009

In 2009, there will be an estimated 8,000 new cases of testicular cancer resulting in approximately 350 deaths in the United States. For reasons that remain unclear, the incidence of testicular cancer, the most common solid malignancy in men between the ages of 20 and 35 years, is increasing in both the United States and Europe.

Given the dramatic improvements in survival rates from approximately $60 \%$ in the 1960 s to over $90 \%$ at present, the management of testicular cancer has come to represent a model of the successful multidisciplinary approach to solid tumors. Although testicular cancer is highly curable, it requires appropriate management for patients of all stages, because both the cure rates and morbidity are highly sensitive to nuances in treatment.

The treatment of patients with testicular cancer has evolved substantially over the past 30 years, largely due to the ability of cisplatin-containing combination chemotherapy to cure advanced disease. Chemotherapy regimens in patients with advanced disease are tailored according to prognostic criteria and the IGCCCG risk classification (1998) represents a milestone in international collaboration. Furthermore, the TNM classification has been expanded to incorporate prognostically relevant serum tumor markers. Consequently, the TNMS classification system has been tailored for the use in patient management based on the prognostic variable analyses from both surgical and chemotherapy studies.

Given the high probability of achieving cure, considerable effort has appropriately been focused on individualizing therapy for specific patients in an attempt to minimize morbidity and preserve quality of life without adversely impacting survival. In clinical stage I patients, two examples of this approach include the evolution of surveillance protocols in both seminoma and NSGCT, particularly in low-risk patients; and examining one cycle, rather than two cycles of adjuvant chemotherapy in high-risk patients.

The survival of patients with testicular cancer has identified significant issues that have been underappreciated in the past such as late relapse and the long-term sequelae of serial imaging and treatment, particularly radiation therapy and chemotherapy. Some of these problems have prompted investigators to re-evaluate and challenge previously accepted treatment paradigms such as modified ipsilateral surgical templates in both primary and post chemotherapy RPLNDs.

I would like to thank Dr. Dan Theodorescu for inviting me to be the guest editor for this issue of the World Journal of Urology. I would also like to thank Dr. Thomas Herrmann and his editorial staff for providing invaluable assistance throughout the process. Finally, I am very grateful to the exceptional group of authors for their significant effort and for sharing their insight and expertise in the constantly changing world of testicular cancer. I trust that the readers of World Journal of Urology will reap the obvious benefits of their efforts.
J. Sheinfeld $(\square)$

Department of Surgery,

Memorial Sloan-Kettering Cancer Center, New York, USA

e-mail: sheinfej@mskcc.org 